\title{
Effect of Two Passive Stretch Manoeuvres for 4 Weeks on Pectoralis Minor Length and Scapular Kinematics among Collegiate Swimmer
}

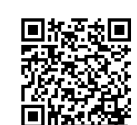

\author{
Arpit Kamboj ${ }^{1}$, Saleem Akhtar Naqvi², Aashish Jain ${ }^{3}$ and Madhusudan Tiwari ${ }^{4 *}$ \\ ${ }^{1} M P T$ (SPORTS), India \\ ${ }^{2}$ Dean \& Principal, Faculty of Physiotherapy, India
}

${ }^{3}$ Assistant Professor, Faculty of Physiotherapy, India

${ }^{4}$ Associate Professor, Faculty of Physiotherapy, Sankalchand Patel University, India

Submission: May 23, 2018; Published: July 18, 2018

*Corresponding author: Madhusudan Tiwari, Associate Professor \& In charge Dean, Faculty of Physiotherapy, Sankalchand Patel University, Visnagar, Gujarat, India, Email: drmstiwari@rediffmail.com

\begin{abstract}
Purpose of the study: Purpose of this study was to compare the acute effects of two passive stretch maneuvers aimed at lengthening the pectoralis minor and the subsequent effects on scapular kinematics among a group of collegiate swimmers.

Study design: Experimental study.

Methodology: A convenience sample of 30 swimmers subject divided into two group, Group B and Group A who fulfilled the inclusion criteria were included in the study. Testing occurred during a single session at the participating team's swimming facilities or within a biomechanics using a pre-test post-test design. Each shoulder was randomly assigned as either focused stretch shoulder, gross stretch shoulder, or control shoulder. Scapular kinematic data for the Group B- experimental shoulders (focused stretch, gross stretch) were measured immediately before and after an application of one of the two passive stretch treatments. Group A- Control shoulders received initial scapular kinematic measurements and post-intervention measurements after an approximate 1.5 minute rest period after performing 5 repetition of each movement of Active Range of Motion exercise of Shoulder. This rest period was approximately the same amount of time necessary to apply the assigned stretch to the experimental shoulders. After the collection of demographic data, a detailed tightness of pectoralis minor was measured by inch tape method. The measurement was taken by standing the participant erect with resting his arm at their side. The distance was taken between origin and insertion of the muscle. The scapular protraction was measured in three position of the shoulder joint i.e., $0^{\circ}, 45^{\circ}$, and $90^{\circ}$. The lateral part of calliper was placed on top of inferior angle and medial part of scapula was placed on corresponding spinous process. The scapular measurement for maximum abduction was measured with gravity inclinometer, the device was placed in the root of spine of scapula. This position was established so that when subjects elevate his arm, their arm were raised in the scapular plane ( $40^{\circ}$ anterior to the frontal plane) while maintaining the contact with the guide pole. The measurement is taken in the maximum elevation of the arm.
\end{abstract}

Results: After measurement of the pectoralis minor length the mean in 0 week for Group A (X=7.56), Group B (X=8.8,) and in $4^{\text {th }}$ week for Group A (X=7.66), Group B (X=9.65). The mean of ROTATION 90 UPWARDS 0 week for Group A (X=15), Group B (X=11.9) mean of ROTATION 90 UPWARDS $4^{\text {th }}$ week for Group A (X=16.33), Group B (X=15.46) mean of SCAPULAR PROTRACTION 0 week for Group A (X=8.38), Group B (X=8.162) mean of SCAPULAR PROTRACTION $4^{\text {th }}$ week for Group A (X=8.48), Group B (X=8.54) With $t$-value and p-value of pcetoralis minor length $\mathrm{cm}$ on pre $\left(0\right.$ week) is $\mathrm{t}=0.02476$ and $(\mathrm{p}>0.05) \mathrm{t}$-value and $\mathrm{p}$-value after $4^{\text {th }}$ week is $\mathrm{t}=3.275$ and $(\mathrm{p}<0.05)$ with $\mathrm{t}$-value and $\mathrm{p}$-value of ROTATION 90 UPWARDS on pre (o week) is $\mathrm{t}=0.04057$ and ( $p>0.05) \mathrm{t}$-value and $\mathrm{p}$-value after $4^{\text {th }}$ week is $\mathrm{t}=1.3096$ and $(\mathrm{p}<0.05)$ with $\mathrm{t}$-value and $\mathrm{p}$-value of SCAPULAR PROTRACTION on pre $\left(1^{\text {st }}\right.$ week) is $\mathrm{t}=0.00276$ and $(\mathrm{p}>0.05) \mathrm{t}$-value and $\mathrm{p}$-value after $4^{\text {th }}$ week is $\mathrm{t}=0.0034$ and $(\mathrm{p}<0.05)$. Also the graphical representation of data clearly shows that as compared to group A Group B has significant difference in (pre-post session) in all three variables. Upon intergroup analysis this shows that Group B has shown significant improvement than that of Group A.

Conclusion: Passive stretch manoeuvre are effective in maintenance of conditioning of pectoralis minor muscles and scapular kinematics among collegiate swimmers

Keywords: Pectoralis minor tightness; Scapular upward rotation and protraction; Scapular kinematics

\section{Introduction}

Competitive swimmers routinely swim six to seven days per week during the competitive season, reaching distances of six to eight miles per practice, which equates to approximately 2500 sequential shoulder revolutions per day [1]. As such, the 
scapular orientations and kinematics during humeral elevation tasks of swimmers can be altered due to changes of surrounding musculature including weakness, fatigue, and inflexibility [2]. Optimal shoulder function is closely associated with proper scapular orientation and motion [2-5]. Therefore, it is vital that any alterations to the periscapular musculature are addressed through preventative and rehabilitative interventions among such athletes.

Scapular dyskinesis has been associated with several shoulder pathologies, such as subacromial impingement syndrome [6-9], rotator cuff pathology [10-12], internal impingement [13], glenohumeral instability [14,15], and adhesive capsulitis [16-18]. One specific cause of scapular dyskinesis may be an abnormally shortened pectoralis minor muscle $[8,19]$. Diminished pectoralis minor flexibility prohibits optimal scapular kinematics, specifically in upward rotation, external rotation, and posterior tilting [20,21]. Therefore, tightness of the pectoralis minor may be a contributing factor to various shoulder injuries, most frequently, subacromial impingement syndrome $[6,8,22]$. Furthermore, tightness of the pectoralis minor may be more prevalent among swimmers due to the repetitive overhead nature of their sport [23].

Due to the potential for development of scapular dyskinesia and upper extremity injury when pectoralis minor tightness is present, stretching of the pectoralis minor muscle is believed to be critical in the prevention and treatment of shoulder dysfunction. Various stretching techniques to lengthen the pectoralis minor are currently used in clinical practice $[19,21]$. However, results of studies that have investigated optimal stretching techniques for the pectoralis minor are conflicting, which has caused increased confusion among researchers and clinicians [20,21,24-26].

Further investigations are necessary to determine how stretching exercises alone might acutely affect the length of the pectoralis minor, as well as the dynamic orientation of the scapula. Therefore, the purpose of this study is to compare the acute effects of two passive stretch maneuvers aimed at lengthening the pectoralis minor and the subsequent effects on scapular kinematics among a group of collegiate swimmers. The authors hypothesized that the passive stretching maneuvers utilized in this study would acutely change the length of the pectoralis minor muscle and affect scapular kinematics among a sample of collegiate swimmers to provide optimal congruence between the glenoid and the humeral head. The shoulder joint enjoy mobility at the cost of stability with respect to bony articularion. The shoulder complex is highly dependent upon soft tissue relationship to maintain joint congruency.

The knowledge of scapula kinematic function and evaluation is growing and understanding the role of scapula in shoulder function and dysfunction is evolving. Movement at the glenohumeral joint can take place in all directions: flexion and extension; abduction and adduction; internal and external rotation; and circumduction.

Each movement is brought about by different groups of muscles. Rotation if the humerus is accomplished by a group of four muscles, subcapularis, supraspinatus, infraspinatus and teres minor collectively called rotator cuff. These muscles originate on different part of scapula, insert like a cuff around the perimeter of the humeral head, where the tendon blend with the joint capsule. In addition to externally and internally rotating the humerus, the rotator cuff helps stabilize the joint during abduction by pulling the humeral head into the glenoid fossa.

Scapular rotation is accomplished by the trapezius and serratus anterior muscles. Trapezius originates on the clavicle and the thoracic spine and inserts on the scapula. Scapular muscles for swimmer are the middle and lower serratus anterior muscles produce scapular upward rotations, posterior tilting and external rotation. Upper trapezius assists in medial stabilization and prevention of excess superior translation of the humeral head, as well as production of glenohumeral external rotation during arm elevation. It is important to assess the difference in static scapular position among swimmers to better understand the exact biomechanical changes in sports like swimming.

\section{Methodology}

Thirty healthy swimming athletes (age $=22.13+2.16$ years, height $=171.6 \mathrm{~cm} \pm 6.38 \mathrm{~cm}$, weight $=65.13 \pm 6.56 \mathrm{~kg}$ ) participated in the study. All participants were in season at the time of data collection. Both left and right shoulders of each subject were used for data collection. Shoulders of swimmers that had a recent history (past 2 months) of upper extremity injury or any history of upper extremity surgery were excluded from the study. Total of the 38 subjects, 8 shoulders were excluded from analysis due to previous injury leaving 30 shoulders for final data analysis; Group A- control shoulders ( $n=15)$, Group B- focused stretch shoulders, gross stretch shoulders $(n=15)$.

\section{Instrumentation}

A standard tape measure was used to measure pectoralis minor length. A gravity inclinometer was used to measure scapular kinematics, Scapular protraction was measured by verniercaliper.

\section{Procedure}

Testing occurred during a single session at the participating team's swimming facilities or within a -biomechanics using a pre-test post-test design. Each shoulder was randomly assigned as either focused stretch shoulder, gross stretch shoulder, or control shoulder. All subjects read and signed an informed consent form approved by the institutional review board prior to participation. The study was conducted in accordance with the Helsinki declaration. Scapular kinematic data for the Group B- experimental shoulders (focused stretch, gross stretch) were measured immediately before and after an application of one of 
the two passive stretch treatments. Group A- Control shoulders received initial scapular kinematic measurements and postintervention measurements after an approximate 1.5 minute rest period after performing 5 repetition of each movement of Active Range of Motion exercise of Shoulder. This rest period was approximately the same amount of time necessary to apply the assigned stretch to the experimental shoulders.

\section{Pectoralis minor length measurement}

To measure the length of the pectoralis minor participants were standing erect with their test arm resting at their side. A tape measure was used to measure the linear distance between the origin and insertion of the muscle. The primary investigator of the study performed all measurements. The origin was defined as the inferior aspect of the 4th rib, which was one finger width lateral to the sternum, just lateral to the sternocostal junction. The insertion was defined as the medial-inferior aspect of the coracoid process. This method of measuring pectoralis minor length has previously been proven to be valid intraclass correlation coefficients between 0.82 to 0.87 when compared with measurements made using an electromagnetic tracking system [27].

\section{Scapular kinematic measurement}

The measurement is to be conducted in swimmers statis scapular position. After a verbal description of the measurement procedure, the method is to be demonstrated to the subject. The measurement protocol utilized in this study for scapular upward rotation describe by Johnson et al. [13]. To measure the scapular upward rotation the root of the scapular spine will be identified and marked in rest. The left edge of the gravity inclinometer will be placed on this mark, and another mark will be placed where the right edge of the inclinometer rest on the scapula. These mark ensure that the gravity inclinometer rested on the same location on the scapula in repeated measures. Than the glenohumeral joint is elevated, a vertical guided pole will be secure to the plinth, the standing position of the subjects will be standardized by lines marked on the floor. The position will be established so that when subjects elevates his arm, their arm would raise in the scaplular plane (400 anterior to the frontal plane) while maintaining the contact with the guide pole. The measurement/reading is taken in the maximum elevation of the arm [28-31].

\section{Scapular protraction measurement}

Scapular protraction measurement will be taken with the participant standing with normal relaxed posture the measurement will be performed at three positions $(00,450$, 900) hands on hips and 900 of glenohumeral abduction with maximum internal rotation. First the inferior angle of scapula was palpated and lateral arm of the calliper was placed at the top of the inferior angle. The medial arm of the calliper will be positioned at the corresponding spinous process, and the measurement was recorded. This process was repeated three times and the measurement was taken.

\section{Pectoralis minor stretches}

It is accepted that a passive stretch duration of 15 to 30 seconds and a frequency of 2 to 4 repetitions is appropriate for acutely improving flexibility $[32,33]$. Thus, the focused and gross passive stretching interventions in this study were performed for two sequential repetitions, holding the stretches for 30 seconds, with a 30 second break between each stretch. The primary investigator applied all stretches at the end range of motion. For the focused stretch shoulders, the authors placed each subject in a supine position with the test arm at their side while the primary investigator palpated medially into the proximal axilla, followed by proceeding superiorly towards the coracoid process. This maneuver allowed the investigator's fingers to be fixed posterior to the proximal end of the pectoralis minor muscle. The investigator then applied pressure in the anterior direction, similar to attempting to lift the muscle, thereby applying tensile force directly to the pectoralis minor. The opposite hand of the investigator was used to stabilize the scapula and humeral head. For the gross-stretch, subjects were positioned in the supine position with the test arm abducted and externally rotated to $90^{\circ}$ and the elbow flexed to $90^{\circ}$. The investigator stabilized each subject's body by placing a hand on the contralateral coracoid. The investigator then passively, horizontally abducted the subject's shoulder.

\section{Statistical Analyses}

A Paired $\mathrm{T}$ test with repeated measures on time was utilized for comparison. Time (pre-, post-test) and shoulder assignment (control, focused stretch, gross stretch) were the independent variables. The dependent variables included a linear measurement of pectoralis minor length, as well as angular measurements of scapular upward/downward rotation, external/internal rotation, and anterior/posterior tilting. If a significant group-by-time interaction was found. Withingroup effect size was calculated as (post-intervention mean -pre-intervention mean)/pre-intervention standard deviation. The effect size calculations provide an indication of clinical meaningfulness of changes among the dependent variables. Alpha level was set a priori at 0.05 . All data were analyzed using SPSS software (Version 21.0, IBM Corp).

\section{Result}

Descriptive data for pre- and post-test pectoralis minor length and scapular kinematics of the two groups are presented in the Graphs. Pectoralis minor length showed a significant interaction effect $(p=0.0028)$. The effect size suggests that a small to moderate amount of the change in pectoralis minor length may be attributed to the treatment (Figure 1-6).

\section{Discussion}

Williams et al. [34] did the study on acute effect of two passive stretch maneuvers on pectoralis minor length and scapular kinematics among colligate swimmer. They found that the gross stretch shoulders had a significant increase in pectoralis minor 


\section{Journal of Yoga and Physiotherapy}

length compared to the control shoulders $(\mathrm{P}=0.007)$. There were no other significant changes in length for either the focused stretch or control shoulders ( $\mathrm{P}>0.07)$. No statistically significant
$(\mathrm{P}>0.08)$ differences for all three scapular kinematic variables were found among any of the three groups $(\mathrm{P}>0.08)$.

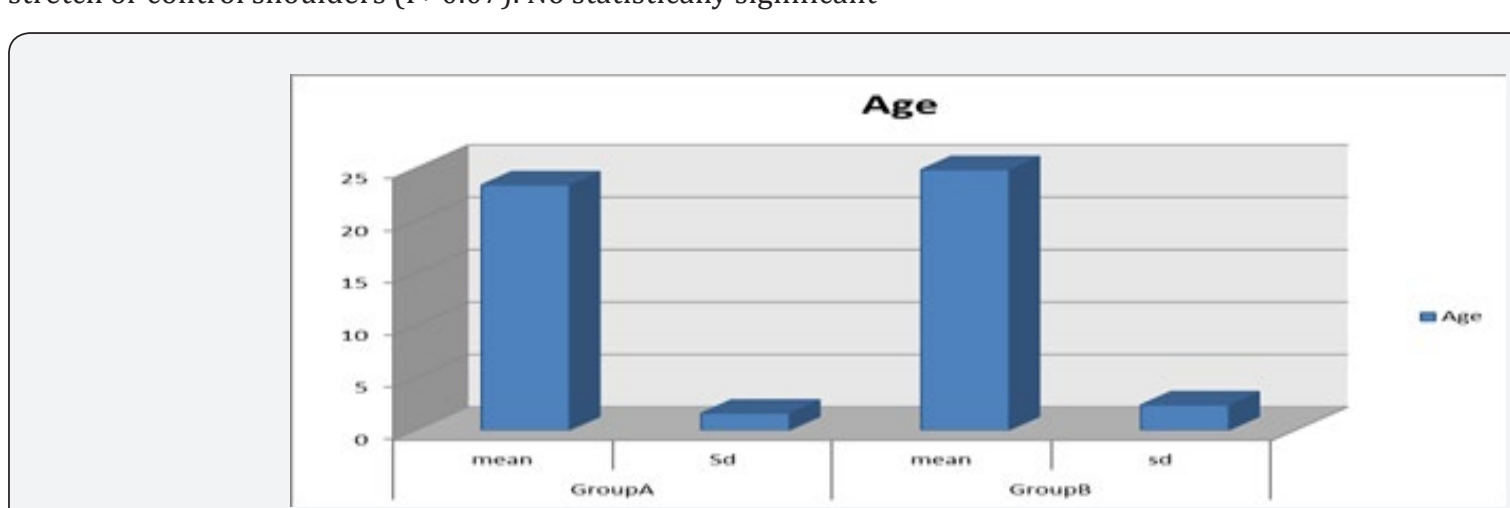

Figure 1: Comparison of mean value and standard deviation for age between Group A and Group B.

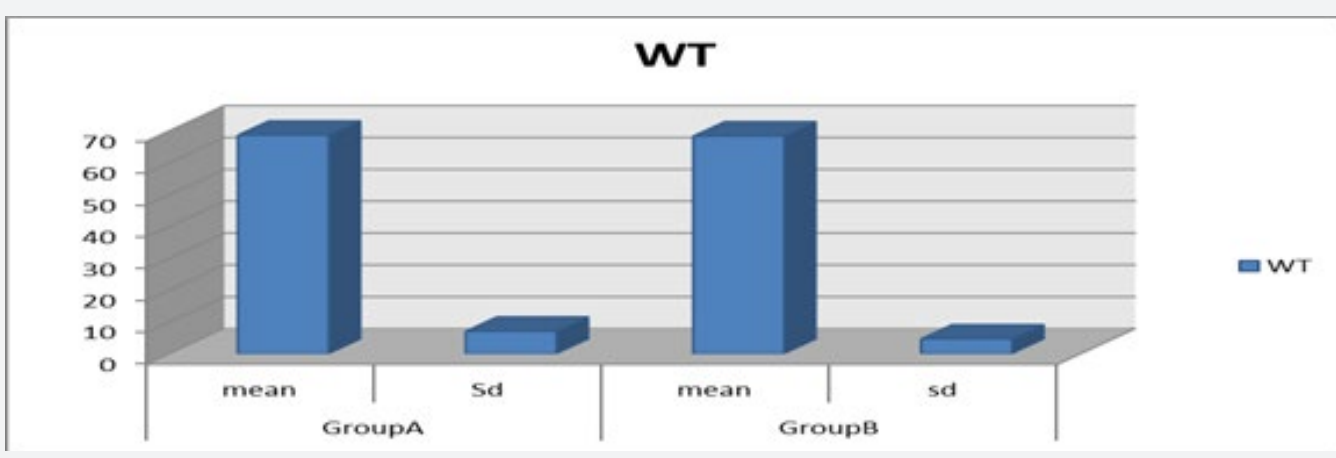

Figure 2: Comparison of mean value and standard deviation for weight between Group A and Group B.
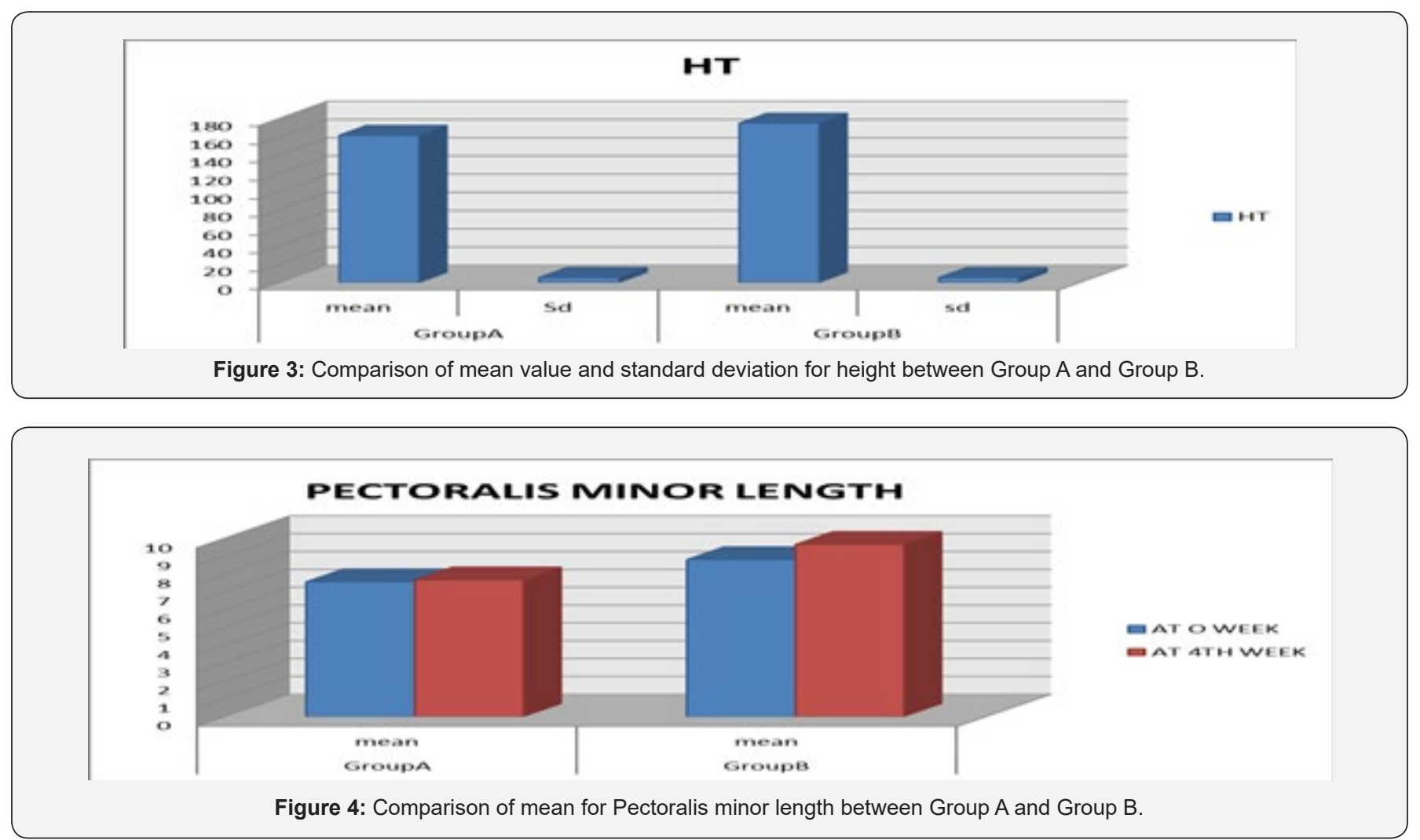

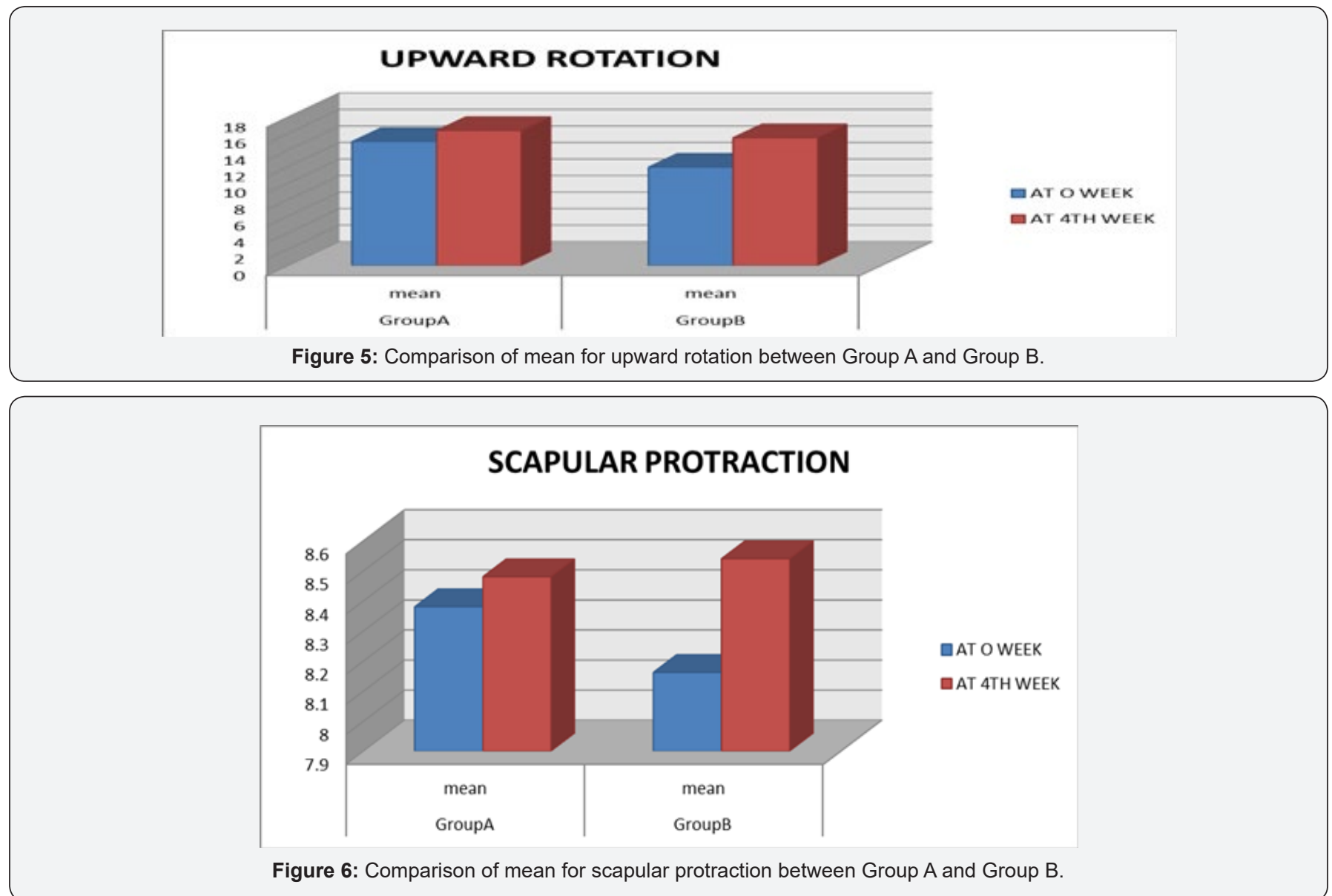

Purpose of our study was to compare the acute effects of two passive stretch maneuvers aimed at lengthening the pectoralis minor and the subsequent effects on scapular kinematics among a group of collegiate swimmers. The total participants were 30 (15 in Group A and 15 in Group B). The analysis of mean and standard Deviation (S.D) of age for Group A (X=23.4, SD=1.63), Group B (X=24.86, $\mathrm{SD}=2.416)$. The analysis of mean and standard Deviation (S.D) of wt for Group A (X=68.6, SD=7.05), Group $B(X=68.33, S D=4.639)$. The analysis of mean and standard Deviation (S.D) of ht for Group A (X=161.66, SD=5.58), Group $\mathrm{B}(\mathrm{X}=174.86, \mathrm{SD}=5.566)$. The analysis of mean and standard Deviation (S.D) of PECTORALIS MINOR LENGTH cm IN 0 week for Group A ( $X=7.56, S D=0.59)$, Group B ( $X=8.8, S D=1.146)$. The analysis of mean and standard Deviation (S.D) of PECTORALIS MINOR LENGTH $\mathrm{cm}$ in 4th week for Group A (X=7.66, SD=0.58), Group B (X=9.65, SD=0.97). The analysis of mean and standard Deviation (S.D) of ROTATION 90 UPWARDS 0 week for Group A $(X=15, S D=6.26)$, Group B $(X=11.9, S D=5.312)$. The analysis of mean and standard Deviation (S.D) of ROTATION 90 UPWARDS 4th week for Group A ( $X=16.33, S D=5.97)$, Group B ( $X=15.46$, $\mathrm{SD}=4.824$. The analysis of mean and standard Deviation (S.D) of SCAPULAR PROTRACTION 0 week for Group A (X=8.38, $S D=5.51)$, Group $B(X=8.162, S D=0.67)$. The analysis of mean and standard Deviation(S.D) of SCAPULAR PROTRACTION 4th week for Group A (X=8.48, SD=5.48), Group B (X=8.54, SD=0.66) With $\mathrm{t}$-value and $\mathrm{p}$-value of pectoralis minor length $\mathrm{cm}$ on pre ( 0 week) is $\mathrm{t}=0.02476$ and $(\mathrm{p}>0.05) \mathrm{t}$-value and $\mathrm{p}$-value after 4 th week is $t=3.275$ and $(p<0.05)$ with $t$-value and $p$-value of ROTATION 90 UPWARDS on pre (o week) is $t=0.04057$ and $(p>0.05) t$-value and $\mathrm{p}$-value after 4 th week is $\mathrm{t}=1.3096$ and $(\mathrm{p}<0.05)$ With $\mathrm{t}$-value and $p$-value of SCAPULAR PRET on pre(1st week) is $t=0.00276$ and $(p>0.05) t$-value and $p$-value after 4 th week is $t=0.0034$ and $(\mathrm{p}<0.05)$.

Also the graphical representation of data clearly shows that as compared to group A Group B has significant difference in (pre-post session) in all three variable. This shows that Group B has shown significant improvement than that of Group A. Borstad \& Ludewig [24] Compared three pectoralis minor stretches. Although, all three stretches were reported to increase the length of the pectoralis minor during the stretch period, the "unilateral self-stretch" was found to create the most tissue lengthening. This stretch was performed in a standing position with the target shoulder abducted to $90^{\circ}$ and the elbow flexed to $90^{\circ}$. With the hand's volar surface of the target arm placed against a vertical door frame or other flat rigid structure the subject then rotated their trunk away from the target shoulder.

The gross stretch performed in our investigation was similar to Borstad \& Ludewig's [24] self-stretch as both stretching maneuvers place the humerus in an abducted and externally 
rotated position while stretching the shoulder further into horizontal abduction and sequentially retracting the scapula. Similar to Borstad \& Ludewig's [24] findings, the gross stretch procedure in the current study showed an increase in the length of the pectoralis minor compared to control shoulders. This shoulder position is in accordance with other investigators who suggest that applying a stretch to the pectoralis minor would require movement of the muscle's insertion in a posterior direction in conjunction with scapular retraction that is performed at or above $30^{\circ}$ of flexion or elevation in the scapular plane (scaption), thereby lengthening the muscle. The authors specifically chose to perform the gross stretch with the subjects lying supine as it allowed the investigator to better control the amount of overpressure applied during the stretches. Subjects performing the stretch independently in a standing position may demonstrate varying amounts of applied overpressure to the shoulder during the stretch. Wang et al. [26] provided subjects with a home exercise program that incorporated gross strengthening of the shoulder complex as well as a stretching procedure for the pectoralis minor over a six week period. A corner stretching exercise, similar to the gross stretch used in the current research, was performed with the subjects' hands above his or her head on adjoining corner walls. The subjects leaned into the wall in order to feel an anterior chest and shoulder stretch and hold for duration of ten seconds and ten repetitions. The researchers' results revealed significantly less scapular upward rotation and greater internal rotation and inferior translation of the scapula at a horizontal arm position following the exercise program. However, McClure et al. [34]. prescribed a six-week home exercise program to subjects diagnosed with subacromial impingement syndrome using a similar self stretch and reported no significant kinematic changes.

\section{Conclusion}

Passive stretch manoeuvre are effective in maintenance of conditioning of pectoralis minor muscles and scapular kinematics among collegiate swimmers.

\section{Limitation}

Hand function is as important as shoulder function in swimmer but hand function is not assessed in this study. Neck extensor is also in a constant isometric contraction along with protectors of shoulder. The study would be more significant if we would have considered neck movement along with shoulder movement.

\section{References}

1. Pink MM, Tibone JE (2000) The painful shoulder in the swimming athlete. Orthop Clin North Am 31(2): 247-261.

2. Kibler WB (2006) Scapular involvement in impingement: signs and symptoms. Instr Course Lect 55: 35-43.

3. Kibler WB, McMullen J (2003) Scapular dyskinesis and its relation to shoulder pain. J Am Acad Orthop Surg 11(2): 142-151.

4. McClure PW, Michener LA, Sennett BJ, Karduna AR (2001) Direct 3-dimensional measurement of scapular kinematics during dynamic movements in vivo. J Shoulder Elbow Surg 10(3): 269-277.

5. Meyer KE, Saether EE, Soiney EK, Shebeck MS, Paddock KL, et al. (2008) Three-dimensional scapular kinematics during the throwing motion. J Appl Biomech 24(1): 24-34.

6. Lukasiewicz AC, McClure P, Michener L, Pratt N, Sennett B (1999) Comparison of 3-dimensional scapular position and orientation between subjects with and without shoulder impingement. J Orthop Sports Phys Ther 29(10): 574-583.

7. Su KP, Johnson MP, Gracely EJ, Karduna AR (2004) Scapular rotation in swimmers with and without impingement syndrome: Practice effects. Med Sci Sports Exerc 36(7): 1117-1123.

8. Ludewig PM, Cook TM (2000) Alterations in shoulder kinematics and associated muscle activity in people with symptoms of shoulder impingement. Phys Ther 80(3): 276-291.

9. Atalar H (2009) Restricted scapular mobility during arm abduction: implications for impingement syndrome. Acta Orthop Belg 75(1): 1924.

10. Graichen H, Stammberger T, Bonel H, Wiedemann E, Englmeier $\mathrm{KH}$, et al. (2001) Three-dimensional analysis of shoulder girdle and supraspinatus motion patterns in patients with impingement syndrome. J Orthop Res 19(6): 1192-1198.

11. Mell AG, LaScalza S, Guffey P, Ray J, Maciejewski M, et al. (2005) Effect of rotator cuff pathology on shoulder rhythm. J Shoulder Elbow Surg 14(1 Suppl S): 58S-64S.

12. Yamaguchi K, Sher JS, Andersen WK, Garretson R, Uribe JW, et al. (2000) Glenohumeral motion in patients with rotator cuff tears: A comparison of asymptomatic and symptomatic shoulders. J Shoulder Elbow Surg 9(1): 6-11.

13. Laudner KG, Myers JB, Pasquale MR, Bradley JP, Lephart SM (2006) Scapular dysfunction in throwers with pathologic internal impingement. J Orthop Sports Phys Ther 36(7): 485-494.

14. Ogston JB, Ludewig PM (2007) Differences in 3-dimensional shoulder kinematics between persons with multidirectional instability and asymptomatic controls. Am J Sports Med 35(8): 1361-1370.

15. Illyes A, Kiss RM (2006) Kinematic and muscle activity characteristics of multidirectional shoulder joint instability during elevation. Knee Surg Sports Traumatol Arthrosc 14(7): 673-685.

16. Fayad F, Roby Brami A, Yazbeck C, Hanneton S, Lefevre Colau MM, et al. (2008) Three-dimensional scapular kinematics and scapulohumeral rhythm in patients with glenohumeral osteoarthritis or frozen shoulder. J Biomech 41(2): 326-332.

17. Rundquist PJ (2007) Alterations in scapular kinematics in subjects with idiopathic loss of shoulder range of motion. J Orthop Sports Phys Ther 37(1): 19-25.

18. Vermeulen HM, Stokdijk M, Eilers PH, Meskers CG, Rozing PM, et al. (2002) Measurement of three dimensional shoulder movement patterns with an electromagnetic tracking device in patients with a frozen shoulder. Ann Rheum Dis 61(2): 115-120.

19. McClure P, Greenberg E, Kareha S (2012) Evaluation and management of scapular dysfunction. Sports Medicine and Arthroscopy Review 20(1): 39-48.

20. Borstad JD, Ludewig PM (2005) The effect of long versus short pectoralis minor resting length on scapular kinematics in healthy individuals. J Orthop Sports Phys Ther 35(4): 227-238.

21. Muraki T, Aoki M, Izumi T, Fujii M, Hidaka E, et al. (2009) Lengthening of the pectoralis minor muscle during passive shoulder motions and stretching techniques: A cadaveric biomechanical study. Phys Ther 89(4): 333-341. 
22. Hebert LJ, Moffet H, McFadyen BJ, Dionne CE (2002) Scapular behavior in shoulder impingement syndrome. Arch Phys Med Rehabil 83(1): 6069.

23. Rupp S, Berninger K, Hopf T (1995) Shoulder problems in high level swimmers-impingement, anterior instability, muscular imbalance? Int J Sports Med 16(8): 557-562.

24. Borstad JD, Ludewig PM (2006) Comparison of three stretches for the pectoralis minor muscle. J Shoulder Elbow Surg 15(3): 324-330.

25. Roddey TS, Olson SL, Grant SE (2002) The effect of pectoralis muscle stretching on the resting position of the scapula in persons with varying degrees of forward head/rounded shoulder posture. Journal of Manual \& Manipulative Therapy 10(3): 124.

26. Wang CH, McClure P, Pratt NE, Nobilini R (1999) Stretching and strengthening exercises: Their effect on three-dimensional scapular kinematics. Arch Phys Med Rehabil 80(8): 923-929.

27. Borstad JD (2008) Measurement of pectoralis minor muscle length: validation and clinical application. J Orthop Sports Phys Ther 38(4) 169-174.

28. Van der Helm FC (2002) A standardized protocol for the description of shoulder motions. International Shoulder Group of the International Society of Biomechanics.
29. An KN, Browne A0, Korinek S, Tanaka S, Morrey BF (1991) Threedimensional kinematics of glenohumeral elevation. J Orthop Res 9(1) 143-149.

30. Barnett ND, Duncan RD, Johnson GR (1999) The measurement of three dimensional scapulohumeral kinematics-a study of reliability. Clin Biomech (Bristol, Avon) 14(4): 287-290.

31. Karduna AR, McClure PW, Michener LA, Sennett B (2001) Dynamic measurements of three-dimensional scapular kinematics: A validation study. J Biomech Eng 123(2): 184-190.

32. Bandy WD, Irion JM (1994) The effect of time on static stretch on the flexibility of the hamstring muscles. Phys Ther 74(9): 845-850.

33. Taylor DC, Dalton JD, Seaber AV, Garrett WE (1990) Viscoelastic properties of muscle-tendon units the biomechanical effects of stretching. Am J Sports Med 18(3): 300-309.

34. McClure PW, Bialker J, Neff N, Williams G, Karduna A, et al. (2004) Shoulder function and 3-dimensional kinematics in people with shoulder impingement syndrome before and after a 6-week exercise program. Phys Ther 84(9): 832-848.

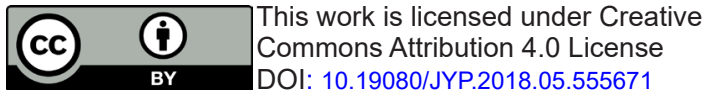

